\title{
Treatment of acne vulgaris in relation to its etiopathogenesis
}

\author{
Florica SANDRU ${ }^{1,2}$, Mihai Cristian DUMITRASCU ${ }^{2,3}$, Ana Maria Alexandra STANESCU ${ }^{2}$, \\ Dan-Nicolae PADURARU ${ }^{2,4}$, Diana SPINU², Adelina POPA ${ }^{2}$ \\ ${ }^{1}$ Department of Dermatology, Elias Emergency University Hospital, Bucharest, Romania \\ 2 "Carol Davila" University of Medicine and Pharmacy, Bucharest, Romania \\ ${ }^{3}$ Department of Obstetrics \& Gynecology, Emergency University Hospital, Bucharest, Romania \\ ${ }^{4}$ Department of Surgery, Emergency University Hospital, Bucharest, Romania
}

\begin{abstract}
Acne vulgaris represent a frequent cutaneous disease manifested by chronic or recurrent development of papules, pustules, or nodules at the level of the face, neck, proximal upper extremities or trunk. The pathogenesis of acne vulgaris includes the interaction of multiple factors that lead to the development of comedones and inflammation. Although acne vulgaris dosen't represent a disease that is physically disabling, its psychologic impact can be striking, contributing to anxiety, low self-esteem and depression. As a result of all these, is a big demand for effective therapies for ane vulgaris.

The pathogenesis and the treatment for each pathogenic cause of acne vulgaris will be reviewed here. Topical retinoids have a good influence for both comedonal (non-inflammatory) and inflammatory acne and should be included in the initial management of most of the patients. The efficacy of topical retinoids for inflammatory acne may be due to a combination of the intrinsic anti-inflammatory properties of the topical retinoids and their ability in the prevention of the formation of microcomedones.
\end{abstract}

Keywords: acne vulgaris, comedones, Cutibacterium acnes, sebaceous glands, topical retinoidsance

\section{INTRODUCTION}

Acne vulgaris represent a frequent cutaneous disease manifested by chronic or recurrent development of papules, pustules, or nodules at the level of the face, neck, proximal upper extremities or trunk. Acne vulgaris represent an inflammatory disorder of the pilosebaceous complex which is formed by the hair follicle and the sebaceous gland.The pathogenesis of acne vulgaris includes the interaction of multiple factors that lead to the development of comedones and inflammation. Although acne vulgaris dosen't represent a disease that is physically disabling, its psychologic impact can be striking, contributing to anxiety, low self-es- teem and depression $(1,2,3)$. As a result of all these, is a big demand for effective therapies for ane vulgaris.

Acne vulgaris is diagnosed most frequent among adolescents and young adults but this is not a rule and this disease is not limited to these ages. The degree of skin involvement varies from an minimal involvement to an disfiguring and highly inflammatory involvement. Scarring, hyperpigmentation, and negative psychosocial effects are the most common complications (4).

The pathogenesis and the treatment for each pathogenic cause of acne vulgaris will be reviewed here. At this moment, quality evidence-based literature in the field of acne is increasing. Medical 
therapies for acne target one or more of four key factors that promote the development of acne lesions (5).

\section{DISCUSSIONS}

Acne vulgaris represent a common disease and occurs most frequently in adolescents populations and young adults. The epidemiological studies estimates that the prevalence of acne vulgaris in adolescents range from $35 \%$ to $90 \%(6,7)$.

The prevalence of acne vulgaris decreases with increasing age. A survey made between the years 2011-2013 of over 1,000 adults in United States found the following rates of self-reported acne in both men and women (8): 20-29 years old - 43$51 \%, 30-39$ years old $-20-35 \%, 40-49$ years old $12-26 \%$ and ages 50 and older $-7-15 \%$.

Acne often begins in pre-adolescent period (712 years) and heals in the third decade, but the disease may persist into adulthood or can develop de novo in adulthood. Adolescent acne exhibits a male predominance, but in contrast, post-adolescent acne predominantly affects the womenas (9).

The pathogenesis of acne vulgaris includes a complex interplay of host factors, represented by androgen-mediated stimulation of sebaceous glands, dysbiosis within the microbiome of the pilosebaceous follicle and innate and cellular immune responses, and it can be influenced by many factors including genetics and, possibly the diet of the individual (10). Knowledge of the mechanisms of action of acne therapies is combined with recognition of specific clinical features to determine the best approach to treatment for each individual.

\section{Lesion development}

The microcomedo - a small, hyperkeratotic plug composed of corneocytes in the lower portion of the follicular infundibulum - is considered to be the precursor for the clinical lesions of acne, which include closed comedones, often colloquially they are called whiteheads, open comedones, often colloquially they are called blackheads, and inflammatory papules, pustules, and nodules. The process by which microcomedones develop and evolve into an other acne lesions remains to be completely elucidated, but it may involve interaction of 4 main pathogenic mechanisms which require specific treatment.

- Follicular hyperproliferation and abnormal desquamation

- Topical retinoids

- Oral retinoids

- Azelaic acid
- Salicylic acid

- Hormonal therapies

- Increased sebum production by sebaceous glands

- Oral isotretinoin

- Hormonal therapies

- Cutibacterium acnes (C. acnes, formerly Propionibacterium acnes) - is an anaerobic diphtheroid that is a normal component of skin flora

- Benzoyl peroxide

- Topical and oral antibiotics

- Azelaic acid

- Inflammation

- Oral isotretinoin

- Oral tetracyclines

- Topical retinoids

- Azelaic acid

The temporal sequence of events leading to formation of acne lesions includes:

- Accumulation of sebum and keratinous material converts a microcomedo into a closed comedo.

- The follicular orifice is opened with continued distension, forming an open comedo. Densely packed keratinocytes, oxidized lipids, and melanin contribute to the dark color of the open comedo.

- Immune responses to $C$. acnes contribute to the development of inflammatory papules and pustules. Follicular rupture releases bacteria, proinflammatory lipids, and keratin into the surrounding dermis, leading to exacerbation of inflammation and/or nodule formation.

\section{Sebaceous glands and the role of androgens}

Androgens contribute to the development of acne by stimulating the growth and secretory function of sebaceous glands, that leads to an increased sebum production. Increased sebum production is thought to provide a medium for the growth of $C$. acnes. $C$. acnes utilizes the triglycerides in sebum as a source of nutrients by hydrolyzing them into glycerol and free fatty acids. The anaerobic environment with an increased composition of lipids in microcomedos allows these bacteria to thrive. Most of the circulating androgens are produced by the gonads and the adrenal gland. Androgen production also occurs within the sebaceous glands, which convert dehydroepiandrosterone sulfate-DHEAS, an adrenal androgen precursor, to testosterone via the action of several enzymes. After that, testosterone is subsequently converted to 5 -alpha-dihydrotestosterone-DHT, via the action of type I 5-alpha-reduc- 
tase in the sebaceous gland. 5-alpha-dihydrotestosterone has greater affinity for androgen receptors than testosterone. Although the majority of patients with acne vulgaris have a normal androgen level, the androgen excess due to several conditions (polycystic ovarian syndrome, congenital adrenal hyperplasia, adrenal or ovarian tumors) can represent a cause of acne vulgaris. In addition, acne typically does not develop prior to adrenarche (the prepubertal period in which levels of dehydroepiandrosterone sulfate rise) with the exception of infantile acne that represents a condition seen in infants that results from excess androgen production by immature adrenal glands or gonads. Men with androgen insensitivity do not produce sebum and do not develop acne (11).

\section{Cutibacterium acnes and inflammation}

C. acnes represents the prominent commensal bacteria within the microbiome of the pilosebaceous follicles has the ability to activate innate and adaptive immune responses, that may contribute to the inflammatory response that is observed in acne vulgaris. Sequencing of the $C$. acnes genome has led to the identification of bacterial properties that can contribute to inflammation and virulence $(12,13,14)$. The acne-associated strains are more probably to carry genes that are associated with antibiotic resistance $(15,16)$. Acne-associated strains also have a greater propensity to stimulate Th 17 cells to secrete interferon-gamma (IFN-gamma) and pro-inflammatory interleukin 17 (IL-17), whereas healthy skin-associated strains stimulate Th 17 cells to produce anti-inflammatory IL-10 $(17,18,19)$. The bacteria C. acnes activate the innate immune response to produce pro-inflammatory IL-1 via the activation of the nod-like receptor P 3 (NLR P3) inflammasome in human sebocytes and monocytes $(20,21)$. It also binds and activates toll-like receptor 2 that is located in the perifollicular macrophages and triggers the release of pro-inflammatory cytokines, including IL-8 and IL-12 $(22,23)$.

The differences in the host inflammatory response to $\mathrm{C}$. acnes or the pathogenicity of specific strains of $C$. acnes that colonize the skin, may contribute to the variation of the prevalence and severity of acne vulgaris (24). C. acnes can also form biofilms within follicles that could contribute to the resistance to therapy (25).

\section{Genetics}

The persons with close family members with acne vulgaris are at increased risk for develop the disorder, supporting a genetic component to the disease (26).

Proposed contributory factors for acne have included skin trauma, dietary habits, stress, insulin resistance, and body mass index.

\section{Associated diseases}

The hyperandrogenism, a disease that may occur in disorders such as late-onset adrenal hyperplasia, polycystic ovarian syndrome and ovarian or adrenal tumors can represent a cause of acne vulgaris. Polycystic ovarian syndrome represents the most common cause of hyperandrogenism in female population (27).

Some examples of rare syndromes that can include acne vulgaris as a feature include SAPHO syndrome - synovitis, acne, pustulosis, hyperostosis and osteitis - and PAPA syndrome - sterile pyogenic arthritis, pyoderma gangrenosum and acne. Patients diagnosticated with SAPHO syndrome exhibit features of inflammatory arthritis or osteitis, particularly at the anterior chest wall level (28).

\section{Acne treatment}

Topical retinoids have a good influence for both comedonal (non-inflammatory) (Fig. 1) and inflammatory acne (Fig. 2) and should be included in the initial management of most of the patients. Topical retinoids are effective in the treatment of comedonal acne due to their ability to normalize follicular hyperkeratosis and prevent formation of the microcomedo, the primary lesion of acne (29). The efficacy of topical retinoids for inflammatory acne may be due to a combination of the intrinsic anti-inflammatory properties of the topical retinoids and their ability in the prevention of the formation of microcomedones $(30,31)$. Topical retinoids can be used as monotherapy in individuals with exclusively comedonal acne.

The patients that have an inflammatory component often benefit from antimicrobial therapies like benzoyl peroxide or other topical antibiotics. Antimicrobial agents reduce the number of proinflammatory $\mathrm{C}$. acnes colonizing the skin. Patients with moderate to severe inflammatory acne often warrant more aggressive treatment with oral antibiotics (32). Antibiotics in the tetracycline class are most frequently used, because they appear to have both antibacterial and anti-inflammatory properties (33). Frequently, practiciens use the combination of benzoyl peroxide with topical or oral antibiotics that decreases the emergence of antibiotic resistant bacteria. Therefore, use of benzoyl peroxide is recommended in patients that are receiving antibiotic therapy $(34,35)$. 


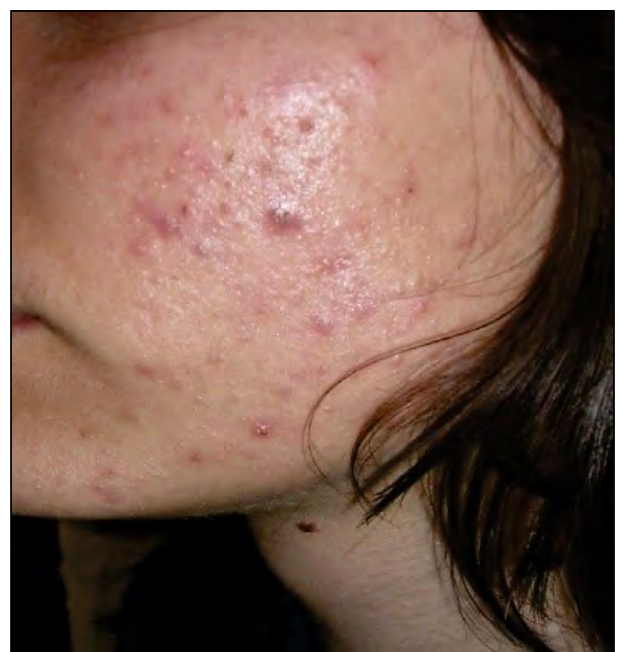

FIGURE 1. Comedonal (non-inflammatory) acne (Personal archive Florica Șandru, MD)

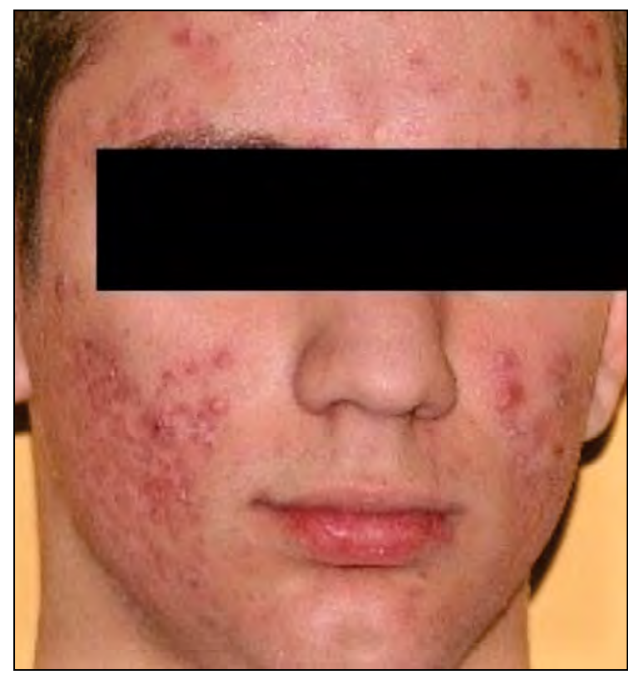

FIGURE 2. Inflammatory acne vulgaris (Personal archive Florica Șandru, MD)

Hormonal therapy may represent the right therapy for women with moderate to severe acne, even in the absence of a hyperandrogenic state.

Patients should be given realistic expectations regarding timelines for improvement. Improvement in acne vulgaris is dependent upon both of the prevention and resolution of acne papules, pustules and nodules. Is often necessary at least two to three months of consistent adherence to a therapeutic regimen prior to concluding that treatment is ineffective. Adjustments in to the therapeutic regimen also may be needed. Acne typically recurs over years, and maintenance therapy is an important component of acne management.

A sample regimen for a patient with mild inflammatory facial acne who is using a topical retinoid, topical benzoyl peroxide, and topical clindamycin is as follows:

- In the morning - The patient need to wash his face with a gentle facial cleanser, then to apply a thin layer of a fixed-dose combination benzoyl peroxide or clindamycin gel to his entire face; an alternative therapeutic regimen could require the patient to wash the face with a benzoyl peroxide cleanser followed by the application of a thin layer of topical clindamycin to the entire face.

- In the evening - The patient need to wash his face with a gentle facial cleanser, then to apply a thin layer of the topical retinoid to his entire face.

\section{CLINICAL CASES}

We will illustrate through two clinical cases the treatment possibilities for acne.

\section{Clinical case 1}

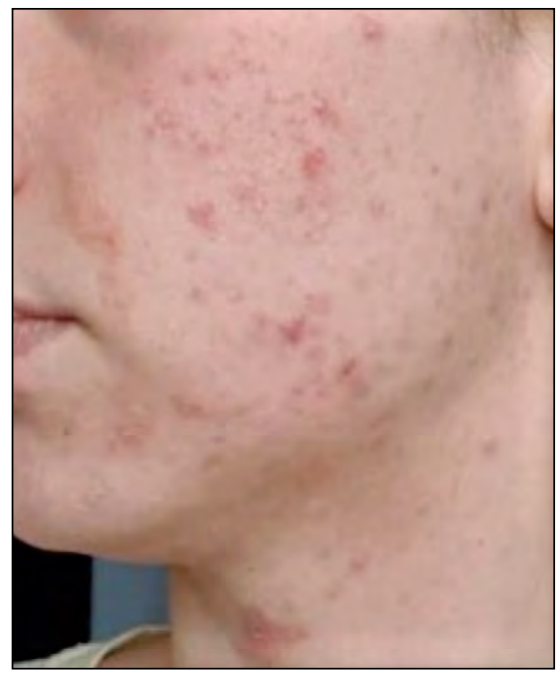

FIGURE 3. $F$, 14 years old, Acne vulgaris, moderate form, in evolution for 2 years, without APP. Previous treatments: antibiotic topical, azelaic acid $=>$ Without a significant improvement (Personal archive Florica Șandru, MD)

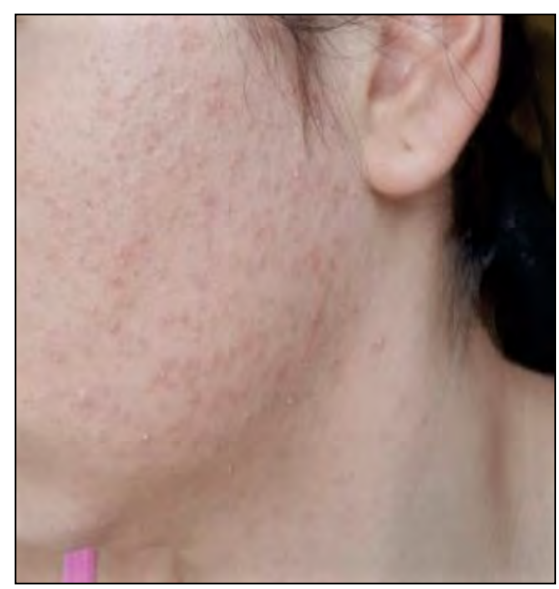

FIGURE 4. Evolution - Current treatment: adapalene $0.1 \%$ - benzoyl peroxide 2.5\%, 1 applic / 2 days, for 4 weeks => Favorable evolution (reducing injuries, reducing inflammation, no associated symptoms) (Personal archive Florica Șandru, MD) 


\section{Clinical case 2}

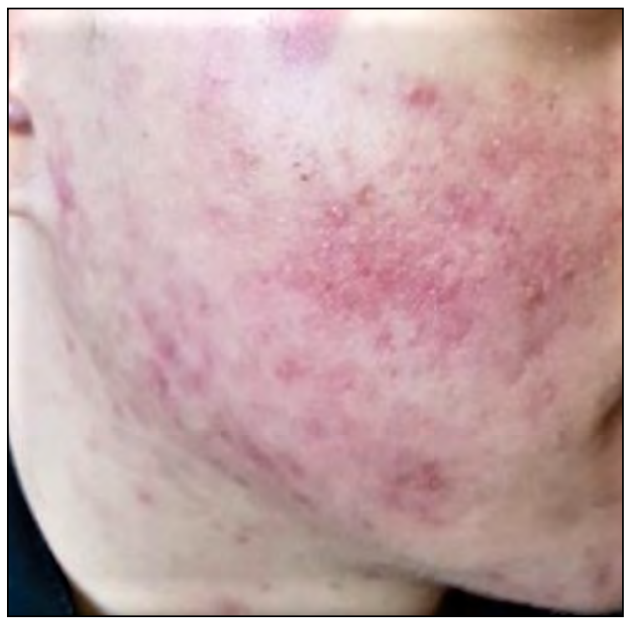

FIGURE 5. $M, 17$ years old, acne vulgaris, moderate to severe, associates erythema, pain sensation, dryness. Previous treatments: local isotretinoin, systemic antibiotic $\Rightarrow$ Without a significant improvement (Personal archive Florica Șandru, MD)

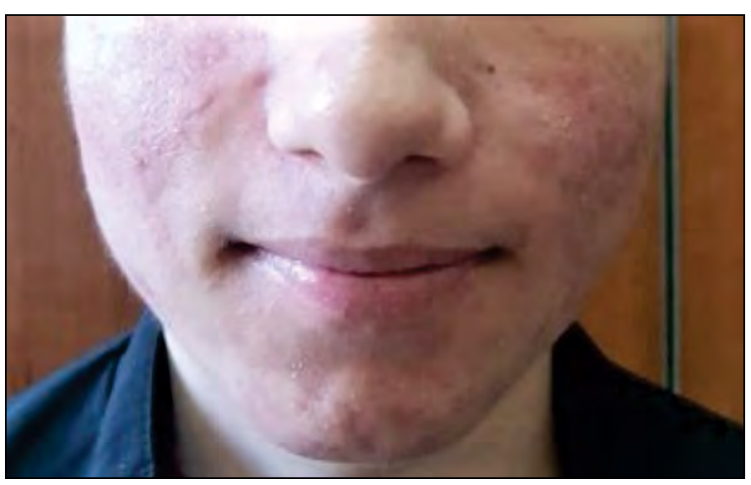

FIGURE 6. Evolution - Current treatment: Adapalene $0.1 \%$ - Benzoyl peroxide 2.5\%, 1 application / 2 days, for 4 weeks; then 1 peak / day, for 8 weeks => Favorable evolution (erythema reduction, blackheads, papulepustules; disappearing symptoms) (Personal archive Florica Șandru, MD)

\section{CONCLUSIONS}

The pathogenesis of acne vulgaris includes the interaction of multiple factors that lead to the de- velopment of comedones and inflammation. Acne vulgaris is diagnosed most frequent among adolescents and young adults but this is not a rule and this disease is not limited to these ages. Medical therapies for acne target one or more of four key factors that promote the development of acne lesions. Knowledge of the mechanisms of action of acne therapies is combined with recognition of specific clinical features to determine the best approach to treatment for each individual.

Androgens contribute to the development of acne by stimulating the growth and secretory function of sebaceous glands, that leads to an increased sebum production. Increased sebum production is thought to provide a medium for the growth of $\mathrm{C}$. acnes. C. acnes utilizes the triglycerides in sebum as a source of nutrients by hydrolyzing them into glycerol and free fatty acids.

Topical retinoids have a good influence for both comedonal (non-inflammatory) and inflammatory acne and should be included in the initial management of most of the patients. Topical retinoids are effective in the treatment of comedonal acne due to their ability to normalize follicular hyperkeratosis and prevent formation of the microcomedo. The efficacy of topical retinoids for inflammatory acne may be due to a combination of the intrinsic anti-inflammatory properties of the topical retinoids and their ability in the prevention of the formation of microcomedones. Topical retinoids can be used as monotherapy in individuals with exclusively comedonal acne. The use of benzoyl peroxide is recommended in patients that are receiving antibiotic therapy. Hormonal therapy may represent the right therapy for women with moderate to severe acne, even in the absence of a hyperandrogenic state. Is often necessary at least two to three months of consistent adherence to a therapeutic regimen prior to concluding that treatment is ineffective.

\section{Conflict of interest: none declared} Financial support: none declared

\section{BIBLIOGRAFIE}

1. Klassen AF, Newton JN, Mallon E. Measuring quality of life in people referred for specialist care of acne: Comparing generic and disease-specific measures. J Am Acad Dermatol 2000; 43:229.

2. Dalgard F, Gieler U, Holm JØ, et al. Self-esteem and body satisfaction among late adolescents with acne: Results from a population survey. J Am Acad Dermatol 2008; 59:746.

3. Yazici K, Baz K, Yazici AE, et al. Diseasespecific quality of life is associated with anxiety and depression in patients with acne. J Eur Acad Dermatol Venereol 2004; 18:435.

4. Lehmann HP, Robinson KA, Andrews JS, et al. Acne therapy: A methodologic review. J Am Acad Dermatol 2002; 47:231.

5. Haider A, Shaw JC. Treatment of acne vulgaris. JAMA 2004; 292:726.

6. Stathakis V, Kilkenny M, Marks R. Descriptive epidemiology of acne vulgaris in the community. Australas J Dermatol 1997; 38:115.
7. Wolkenstein $P$, Machovcová A, Szepietowski JC, et al. Acne prevalence and associations with lifestyle: A crosssectional online survey of adolescents/ young adults in 7 European countries. J Eur Acad Dermatol Venereol 2018; 32:298.

8. Fitz-Gibbon S, Tomida S, Chiu BH, et al. Propionibacterium acnes strain populations in the human skin microbiome associated with acne. J Invest Dermatol 2013; 133:2152. 
9. Goulden V, Clark SM, Cunliffe WJ. Post-adolescent acne: A review of clinical features. Br J Dermatol 1997; 136:66.

10. O'Neill AM, Gallo RL. Host-microbiome interactions and recent progress into understanding the biology of acne vulgaris. Microbiome 2018; 6:177.

11. Imperato-McGinley J, Gautier T, Cai LQ, et al. The androgen control of sebum production. Studies of subjects with dihydrotestosterone deficiency and complete androgen insensitivity. J Clin Endocrinol Metab 1993; 76:524.

12. Grice EA, Kong HH, Conlan $\mathrm{S}$, et al. Topographical and temporal diversity of the human skin microbiome. Science 2009; 324:1190.

13. Fitz-Gibbon S, Tomida S, Chiu BH, et al. Propionibacterium acnes strain populations in the human skin microbiome associated with acne. J Invest Dermatol 2013; 133:2152.

14. Hall JB, Cong Z, Imamura-Kawasawa Y, et al. Isolation and Identification of the Follicular Microbiome: Implications for Acne Research. J Invest Dermatol 2018; 138:2033.

15. Brüggemann $H$, Henne $A$, Hoster $F$, et al. The complete genome sequence of Propionibacterium acnes, a commensal of human skin. Science 2004; 305:671.

16. Brüggemann $\mathrm{H}$. Insights in the pathogenic potential of Propionibacterium acnes from its complete genome. Semin Cutan Med Surg 2005; 24:67.

17. Lomholt HB, Kilian M. Population genetic analysis of Propionibacterium acnes identifies a subpopulation and epidemic clones associated with acne. PLoS One 2010; 5:e12277.

18. Agak GW, Qin M, Nobe J, et al. Propionibacterium acnes Induces an IL-17 Response in Acne Vulgaris that Is
Regulated by Vitamin A and Vitamin D. J Invest Dermatol 2014; 134:366.

19. Agak GW, Kao S, Ouyang K, et al. Phenotype and Antimicrobial Activity of Th17 Cells Induced by Propionibacterium acnes Strains Associated with Healthy and Acne Skin. J Invest Dermatol 2018; 138:316.

20. Li ZJ, Choi DK, Sohn KC, et al. Propionibacterium acnes activates the NLRP3 inflammasome in human sebocytes. J Invest Dermatol 2014; 134:2747.

21. Qin M, Pirouz A, Kim MH, et al. Propionibacterium acnes Induces IL-1 $\beta$ secretion via the NLRP3 inflammasome in human monocytes. J Invest Dermatol 2014; 134:381.

22. Shibata M, Katsuyama M, Onodera T, et al. Glucocorticoids enhance Toll-like receptor 2 expression in human keratinocytes stimulated with Propionibacterium acnes or proinflammatory cytokines. J Invest Dermatol 2009; 129:375.

23. Kim J, Ochoa MT, Krutzik SR, et al. Activation of toll-like receptor 2 in acne triggers inflammatory cytokine responses. J Immunol 2002; 169:1535.

24. Sugisaki $H$, Yamanaka K, Kakeda M, et al. Increased interferon-gamma, interleukin12 p40 and IL-8 production in Propionibacterium acnes-treated peripheral blood mononuclear cells from patient with acne vulgaris: Host response but not bacterial species is the determinant factor of the disease. J Dermatol Sci 2009; 55:47.

25. Jahns AC, Lundskog B, Ganceviciene R, et al. An increased incidence of Propionibacterium acnes biofilms in acne vulgaris: A case-control study. $\mathrm{Br} \mathrm{J}$ Dermatol 2012; 167:50.

26. Trivedi NR, Gilliland KL, Zhao W, et al. Gene array expression profiling in acne lesions reveals marked upregulation of genes involved in inflammation and matrix remodeling. J Invest Dermatol 2006; 126:1071.

27. Ballanger F, Baudry P, N'Guyen JM, et al. Heredity: A prognostic factor for acne. Dermatology 2006; 212:145.

28. Yosipovitch G, Tang M, Dawn AG, et al. Study of psychological stress, sebum production and acne vulgaris in adolescents. Acta Derm Venereol 2007; 87:135.

29. Gollnick H, Cunliffe W, Berson D, et al. Management of acne: A report from a Global Alliance to Improve Outcomes in Acne. J Am Acad Dermatol 2003; 49:S1.

30. Thielitz A, Gollnick H. Topical retinoids in acne vulgaris: Update on efficacy and safety. Am J Clin Dermatol 2008; 9:369.

31. Leyden JJ, Shalita A, Thiboutot D, et al. Topical retinoids in inflammatory acne: $\mathrm{A}$ retrospective, investigator-blinded, vehicle-controlled, photographic assessment. Clin Ther 2005; 27:216.

32. Taglietti M, Hawkins CN, Rao J. Novel topical drug delivery systems and their potential use in acne vulgaris. Skin Therapy Lett 2008; 13:6.

33. Date AA, Naik B, Nagarsenker MS. Novel drug delivery systems: Potential in improving topical delivery of antiacne agents. Skin Pharmacol Physiol 2006; 19:2.

34. Eichenfield LF, Krakowski AC, Piggott C, et al. Evidence-based recommendations for the diagnosis and treatment of pediatric acne. Pediatrics 2013; 131 Suppl 3:S163.

35. Prodea MC, Sandru F, Carpenco E, Gaje PN, Horhat ID, Ceausu AR, Mederle N, Mederle OA. A Rare Sweat Gland Tumour - Immunohistochemical Features. Rev. Chimia (Bucharest) 2019, 70(5)201:18631865. 